\title{
Universal Hall conductance scaling in non-Hermitian Chern insulators
}

\author{
Solofo Groenendijk $\odot$ and Thomas L. Schmidt \\ Department of Physics and Materials Science, University of Luxembourg, L-1511 Luxembourg, Luxembourg \\ Tobias Meng $\odot$ \\ Institute for Theoretical Physics and Würzburg-Dresden Cluster of Excellence ct.qmat, \\ Technische Universität Dresden, 01069 Dresden, Germany
}

(Received 17 September 2020; accepted 22 February 2021; published 1 April 2021)

\begin{abstract}
We investigate the Hall conductance of a two-dimensional Chern insulator coupled to an environment causing gain and loss. Introducing a biorthogonal linear response theory, we show that sufficiently strong gain and loss lead to a characteristic nonanalytical contribution to the Hall conductance. Near its onset, this contribution exhibits a universal power law with a power $3 / 2$ as a function of Dirac mass, chemical potential, and gain strength. Our results pave the way for the study of non-Hermitian topology in fermionic transport experiments.
\end{abstract}

DOI: 10.1103/PhysRevResearch.3.023001

Topology is a universal organizing principle complementing Landau's theory of symmetry-broken phases [1-3]. In Landau's theory, different phases are distinguished by local order parameters. In a similar vein, topologically distinct phases can be discerned by topological invariants. Unlike local order parameters, which take continuous values, topological invariants are quantized [2,4]: they can only change in discrete steps, and often take values in $\mathbb{Z}_{2}$ or $\mathbb{Z}[2,5]$. In theory, topological phases can be identified by discrete outcomes of nonlocal measurements reflecting the topological invariants $[2,4]$. Prototypical examples of topological states are the integer quantum Hall state in a two-dimensional electron system [6], and the closely related two-dimensional Chern insulators $[4,7,8]$. The topological invariant of these states is the (first) Chern number $C \in \mathbb{Z}$. In principle, the Chern number can be experimentally accessed via the Hall conductance $\sigma_{x y}=$ $\left(e^{2} / h\right) C[6,8]$.

The strict mathematical definition of topological invariants typically requires isolated systems of infinite size [9]. As experimental systems with nontrivial topology are neither infinite nor completely decoupled from their environment, it is important to quantify the extent to which they exhibit quantized responses at all. While finite system sizes only lead to exponentially small corrections to topologically quantized response functions $[10,11]$, the impact of an environment and thus a coupling to external degrees of freedom is still an important open problem.

In this paper, we study the prototypical case of a Chern insulator coupled to an environment, and consider the system in a regime where it can be described by an effective non-Hermitian Hamiltonian with loss and gain terms [12-14]. Whereas the amplitude of the Hall conductance in such a non-

Published by the American Physical Society under the terms of the Creative Commons Attribution 4.0 International license. Further distribution of this work must maintain attribution to the author(s) and the published article's title, journal citation, and DOI.
Hermitian Chern insulator ceases to be quantized [15,16], we find that sufficiently strong loss and gain leads to a new type of universality in the Hall response: a nonanalytic dependence of the Hall conductance on the variation of all relevant system parameters with the exponent $3 / 2$.

This behavior of the Hall conductance is somewhat similar to the behavior of the density of states in systems with loss and gain exhibiting so-called exceptional points. At these points in parameter space, several of the eigenvectors of the effective single-particle Hamiltonian coalesce, such that they no longer form a complete basis [13,14,17-19]. In an electronic measurement, exceptional points are reflected in a characteristic non-analytic contribution to the density of states scaling with a universal exponent $1 / 2$ [20]. The nonanalytic contribution to the Hall conductance identified in this work has an equally universal scaling, albeit with the exponent $3 / 2$. While it can appear in a similar parameter regime as required for exceptional points, we show that both phenomena in general occur independently from one another. Our findings thus not only provide a new quantized fingerprint of non-Hermitian physics, but they do so in a transport quantity that is characteristic of a topological system with otherwise nonquantized response functions, namely the non-Hermitian Chern insulator.

We begin by presenting a generic theory of open electronic systems. The starting point is a Hamiltonian describing both the system and its environment. In many cases, the effect of the environment can be taken into account by introducing non-Hermitian terms in an effective system Hamiltonian. Non-Hermitian Hamiltonians already appear at the level of retarded Green's functions with complex selfenergies, $G^{R}(\mathbf{k}, \omega)=\left[\omega-\mathcal{H}_{0}(\mathbf{k})+\Sigma^{R}(\mathbf{k}, \omega)\right]^{-1}$, where $\mathcal{H}_{0}$ is the Bloch Hamiltonian of the isolated system and $\Sigma^{R}$ is the retarded self-energy brought about by its coupling to the environment. This can in turn be used to construct an effective non-Hermitian Bloch Hamiltonian $\mathcal{H}_{\text {nh }}(\mathbf{k}):=$ $\mathcal{H}_{0}(\mathbf{k})-\Sigma^{R}(\mathbf{k}, 0)$. Another avenue for non-Hermitian Hamiltonians is the Lindblad master equation $\partial_{t} \rho=i[\rho, H]+$ $\sum_{n}\left(L_{n} \rho L_{n}^{\dagger}-\left\{L_{n}^{\dagger} L_{n}, \rho\right\} / 2\right)$ that allows one to define a 
non-Hermitian Hamiltonian as $H_{\mathrm{nh}}=H-(i / 2) \sum_{n} L_{n}^{\dagger} L_{n}$. Here, $\rho(t)$ denotes the system density matrix and $L_{n}$ are Lindblad operators describing quantum jumps due to the coupling to the environment. Quite generally, the dynamics of an open system can thus be described by different forms of effective non-Hermitian Hamiltonians with loss and gain terms, at least in suitable approximations [13,21-26].

In contrast to Hermitian Hamiltonians, non-Hermitian Hamiltonians $H_{\text {nh }}$ have distinct right and left eigenvectors for the same eigenvalue, i.e., $H_{\mathrm{nh}}\left|\phi_{m}\right\rangle=\epsilon_{m}\left|\phi_{m}\right\rangle$ and $\left\langle\tilde{\phi}_{m}\right| H_{\mathrm{nh}}=$ $\left\langle\tilde{\phi}_{m}\right| \epsilon_{m}$ with $\left|\phi_{m}\right\rangle \neq\left|\tilde{\phi}_{m}\right\rangle$. Furthermore, right and left eigenvectors are not necessarily mutually orthogonal, i.e., it is possible that $\left\langle\phi_{m} \mid \phi_{n}\right\rangle \neq 0$ and $\left\langle\tilde{\phi}_{m} \mid \tilde{\phi}_{n}\right\rangle \neq 0$ for $m \neq n$. Instead, the algebra known from Hermitian systems carries over to natural combinations of right and left eigenstates. It is, for instance, possible to choose the eigenstates in such a way that $\left\langle\tilde{\phi}_{m} \mid \phi_{n}\right\rangle=\delta_{m n}$ and $\mathbb{1}=\sum_{m}\left|\phi_{m}\right\rangle\left\langle\tilde{\phi}_{m}\right|$ [27]. This immediately raises the question of how objects as basic as expectation values $\langle O\rangle$ of a system operator $O$ should be interpreted. Whether the expectation value is taken with respect to right eigenstates only, left eigenstates only, or a combination of right and left eigenstates depends on the situation that is modeled by the non-Hermitian Hamiltonian. Technically, this question can be condensed into the equation of motion for the density matrix $\rho$ describing the system [28]. Setups described by a Lindblad master equation have short-term dynamics described by only right eigenvectors, while non-Hermitian descriptions of systems with finite lifetimes rather correspond to combining right and left eigenstates in what is known as a "biorthogonal" quantum description.

In this paper, we study electronic transport in a $2 \mathrm{D}$ electronic system with loss and gain. To describe transport in such an open setup, we consider the system coupled to an electric field. Assuming the field to be weak, we study the time evolution of the biorthogonal expectation value of electric current in linear response. Concretely, we analyze a non-Hermitian Chern insulator modelled by the effective Hamiltonian $H_{\mathrm{nh}}=\sum_{\mathbf{k}} \Psi^{\dagger}(\mathbf{k}) \mathcal{H}_{\mathrm{nh}}(\mathbf{k}) \Psi$ (k) with the nonHermitian Bloch Hamiltonian

$$
\begin{aligned}
\mathcal{H}_{\mathrm{nh}}(\mathbf{k})= & k_{x} \sigma_{x}+k_{y} \sigma_{y}+m \sigma_{z}-\mu \sigma_{0} \\
& -i \Gamma_{0} \sigma_{0}-i\left(\Gamma_{x} \sigma_{x}+\Gamma_{y} \sigma_{y}+\Gamma_{z} \sigma_{z}\right) .
\end{aligned}
$$

Here, $\Psi^{\dagger}(\mathbf{k})=\left[c_{\uparrow}^{\dagger}(\mathbf{k}), c_{\downarrow}^{\dagger}(\mathbf{k})\right]$ denotes the spinor of creation operators for electrons with spin $\sigma=\uparrow, \downarrow$ and momentum $\mathbf{k}=\left(k_{x}, k_{y}\right)^{T}$. In the Bloch Hamiltonian, $\sigma_{x, y, z}$ are Pauli matrices, and $\sigma_{0}$ is the identity matrix. Moreover, $m$ is the Dirac mass and $\mu$ the chemical potential. Loss and gain resulting from the coupling to the environment are encoded in the non-Hermitian terms $\Gamma_{0, x, y, z}$ which we approximate as k-independent. Such momentum independent gain-loss parameter describes for instance most situations of trapped cold atoms that are coupled to an environment [29-34]. Similarly, in electronic systems, the momentum dependent parts of the self-energy are in general subleading with respect to the constant part, although there can also be situations in which the momentum dependence of the non-Hermitian terms is important [35]. Without loss of generality, we will assume $\Gamma_{0, x, y, z} \geqslant$
0 . The system experiences net loss if $\Gamma_{0}>|\boldsymbol{\Gamma}|$, whereas one mode has net gain for $\Gamma_{0}<|\boldsymbol{\Gamma}|$; see Appendix B.

A well-known hallmark of non-Hermitian physics is the presence of exceptional points in the complex band structure. The Hamiltonian (1) exhibits exceptional points at momenta satisfying $\sqrt{\left(k_{x}-i \Gamma_{x}\right)^{2}+\left(k_{y}-i \Gamma_{y}\right)^{2}+\left(m-i \Gamma_{z}\right)^{2}}=0$. Recently, similar exceptional points have gained a lot of attention in photonic systems with parity-time (PT) symmetry [36-41]. In these systems, the complex refractive index of the material plays a similar role as the self-energy in electronic systems. One salient feature of non-Hermitian photonic systems is that close to the exceptional points, PT symmetry breaks down and the system exhibits rather peculiar transport properties such as reflectionless unidirectional wave propagation [40-42], loss induced transparency [43], and nonanalytic frequency response in terms of the system parameter [44]. In contrast, the transport properties of fermionic systems combining strong loss and gain with nontrivial topology have not yet been studied in great detail. Fermionic Chern insulators have recently been realized experimentally using fermionic cold atoms in optical lattices [45,46], a class of systems in which gain and loss cannot be entirely avoided. Turning this nuisance into a resource, cold atoms in optical lattices are promising candidates to engineer specific fermionic non-Hermitian Hamiltonians with nontrivial topology.

The characteristic experimental observable of Chern insulators is their Hall conductance, which is quantized in the absence of coupling to an environment. To analyze the fate of the Hall conductance in the presence of loss and gain, we first define the electric current as $\mathbf{j}=\partial_{\mathbf{k}} \mathcal{H}_{\mathrm{nh}}(\mathbf{k})=$ $\partial_{\mathbf{k}} \mathcal{H}_{0}(\mathbf{k})$, where $\mathcal{H}_{0}$ is the unperturbed part of the Bloch Hamiltonian (i.e., the Hamiltonian at vanishing electric field). We then analyze the time evolution of its expectation value $\langle\mathbf{j}\rangle(t)=\left\langle\tilde{\phi}_{0}(t)|\mathbf{j}| \phi_{0}(t)\right\rangle$, where the initial state $\left|\phi_{0}\right\rangle$ is the right eigenstate $\left|\phi_{m}\right\rangle$ with maximal norm of $e^{-\beta \epsilon_{m}}$, whereas $\left|\tilde{\phi}_{0}\right\rangle$ denotes the corresponding left state $(\beta$ is the inverse temperature). Their time evolution follows from the generalized Schrödinger equation for right and left states, $i \partial_{t}|\phi\rangle=H_{\mathrm{nh}}|\phi\rangle$ and $i \partial_{t}|\tilde{\phi}\rangle=H_{\mathrm{nh}}^{\dagger}|\tilde{\phi}\rangle$. Since we allow the non-Hermitian Hamiltonian to describe both loss and gain, we cannot study the time evolution as usual, assuming that the electric field was switched on at a time $t_{0} \rightarrow-\infty$, but instead need to keep $t_{0}$ finite. Using a Lehmann spectral representation, the current $\left\langle j_{x}\right\rangle$ in the $x$ direction at time $t>t_{0}$ due to an electric field $\mathbf{E}=E_{0, y} e^{-i \omega_{0} t} \mathbf{e}_{y}$ with frequency $\omega_{0}$ in the $y$ direction can then in the zero temperature limit $\beta \rightarrow \infty$ be written as (see Appendix A for details)

$$
\begin{aligned}
\left\langle j_{x}\right\rangle(t)= & \frac{E_{0, y}}{i \omega_{0}} e^{-i \omega_{0} t} \sum_{m, n} \frac{1}{Z}\left\langle\tilde{\phi}_{m}\left|j_{x}\right| \phi_{n}\right\rangle\left\langle\tilde{\phi}_{n}\left|j_{y}\right| \phi_{m}\right\rangle \\
& \times \frac{e^{-\beta \epsilon_{m}}-e^{-\beta \epsilon_{n}}}{\omega_{0}+\epsilon_{m}-\epsilon_{n}}\left(1-e^{i\left(\omega_{0}+\epsilon_{m}-\epsilon_{n}\right)\left(t-t_{0}\right)}\right),
\end{aligned}
$$

where the sums are over all eigenstates of the Hamiltonian $\mathcal{H}_{\mathrm{nh}}, \beta$ is the inverse temperature, and $Z=\sum_{n} e^{-\beta \epsilon_{n}}$ is the generalized partition function. The overall behavior of the current, in particular a nonanalytic dependence on system parameters, can be understood as being handed down to Eq. (2) 
from the effective conductance

$$
\sigma_{x y}=\frac{1}{i \omega_{0}} \sum_{m, n} \frac{1}{Z}\left\langle\tilde{\phi}_{m}\left|j_{x}\right| \phi_{n}\right\rangle\left\langle\tilde{\phi}_{n}\left|j_{y}\right| \phi_{m}\right\rangle \frac{e^{-\beta \epsilon_{m}}-e^{-\beta \epsilon_{n}}}{\omega_{0}+\epsilon_{m}-\epsilon_{n}} .
$$

To evaluate this expression, we now proceed by analytic continuation to the complex plane. We start from the current-current correlation function in imaginary time (see Appendix A)

$$
-\operatorname{Tr}^{\prime}\left[e^{-\beta H_{\mathrm{nh}}} T_{\tau} j_{x}(\tau) j_{y}\left(\tau^{\prime}\right)\right],
$$

where the trace of an operator $O$ is defined with biorthogonal states as $\operatorname{Tr}^{\prime} O=\sum_{n}\left\langle\tilde{\phi}_{n}|O| \phi_{n}\right\rangle$. Using a Lehmann representation, it can easily be shown that the Fourier transform of this to Matsubara frequencies exactly corresponds to the conductance $\sigma_{x y}$ identified in biorthogonal perturbation theory. This allows us to formally evaluate the biorthogonal linear response in the familiar language of Matsubara Green's functions $\mathcal{G}$, for which the current takes the form $\mathbf{j}=-\partial_{\mathbf{k}} \mathcal{G}^{-1}$. The full definition of our analytic continuation scheme also includes the complex form of the loss and gain parameters $\Gamma_{i}$. For the case of quasiparticle lifetimes encoded in complex self-energies, the loss parameter has the Matsubara expression $\Gamma_{i}\left(\omega_{n}\right)=\Gamma_{i} \operatorname{sgn}\left(\omega_{n}\right)$. As we are interested in generalizing the framework of Matsubara-based linear-response theory to describe both quasiparticle loss and gain, we include a similar sign function in our continuation of the non-Hermitian Hamiltonian to the complex plane. It is then straightforward to show that the Hall response takes the usual form [47-50]

$$
\sigma_{x y}=\frac{e^{2}}{h} \frac{\varepsilon^{\mu \nu \lambda}}{24 \pi^{2}} \int d^{3} q \operatorname{Tr}\left[\left(\partial_{\mu} \mathcal{G}^{-1}\right) \mathcal{G}\left(\partial_{\nu} \mathcal{G}^{-1}\right) \mathcal{G}\left(\partial_{\lambda} \mathcal{G}^{-1}\right) \mathcal{G}\right],
$$

where $q=\left(k_{x}, k_{y}, i \omega_{n}\right)$ is the three-momentum including the Matsubara frequency $\omega_{n}$ and the trace is over the resulting $2 \times 2$ matrix. The relation between the Chern topological invariant and the system Green's function was first pointed out by Ref. [51] and extended to interacting systems using interacting Green's function by Refs. [52,53].

The quantization of the Hall conductance in the special case of a Hermitian Chern insulator follows quite elegantly from the interpretation of Eq. (5) as a topological winding number. Namely, if $\mathcal{G}(q)$ is a smooth function of $q$, then the integral in Eq. (5) yields the winding number of the Green's function in the space of $2 \times 2$ matrices $G L(\mathbb{C}, 2)$ as $q$ is varied $[48,54,55]$. This integral is also referred to as Pontryagin index in the literature, and appears in the study of Yang-Mills instantons [56], Wess-Zumino-Witten field theories [54,55], and Chern-Simons theories [57]. In contrast, if the Green's function $\mathcal{G}(q)$ is not continuous, the Hall response is generically nonquantized because the integrand in Eq. (5) is not smooth $[16,19,58]$. The quantization of the Hall conductance is already spoiled in the simple case of a Matsubara self-energy $\Sigma^{M}\left(k_{x}, k_{y}, i \omega_{n}\right)=i \operatorname{sgn}\left(\omega_{n}\right) \Sigma_{0} \mathbb{1}_{2}$, describing for example the coupling to an itinerant magnet $[15,16]$, because this selfenergy entails a discontinuous jump of $\mathcal{G}(q)$ across the real axis. A deviation from the quantized conductance can also arise in periodically driven systems where the conductance is related to pumping the charge between the different bands with different occupation probabilities [59].

We now show that while the amplitude of the Hall response of a non-Hermitian Chern insulator is nonuniversal and nonquantized, universal behavior of the Hall conductance with a quantized scaling can still arise if the non-Hermitian part of the Hamiltonian has a nontrivial matrix structure as in Eq. (1), provided one of the two modes exhibits gain. In the following, we focus on gapped systems with $m \neq 0$ and on chemical potentials near the Dirac point, $\mu^{2} \leqslant m^{2}+\Gamma_{0}^{2}$. The general method to compute the Hall conductance of Chern insulators described by Eq. (1) is given in Appendix B.

If we consider the case $\Gamma_{x, y}=0$ but allow for nonzero $\Gamma_{z}$ and $\Gamma_{0}$, the Hall conductance is the sum of a Fermi sea contribution $\sigma_{x y}^{\text {(sea) }}\left(\Gamma_{0}, \Gamma_{z}\right)$ and a Fermi surface contribution $\sigma_{x y}^{\text {(surface) }}\left(\Gamma_{0}, \Gamma_{z}\right)$ [see Eqs. (B15) and (B13) in Appendix B]. The former is quantized in the limit of vanishing net dissipation $\left(\Gamma_{0}=0\right)$ as long as the chemical potential is in the gap $(|\mu|<|m|)$.

The situation changes dramatically if either $\Gamma_{x}$ or $\Gamma_{y}$ is sufficiently large. Since the system has rotational symmetry in the $x-y$ plane, we focus on the case $\Gamma_{y} \neq 0$. For simplicity, we chose $\Gamma_{z}=0$ for this discussion. The conductance can be computed using Eq. (5) and one finds that the Fermi sea contribution consists of two terms [see Eqs. (B18) and (B19) in Appendix B], such that

$$
\sigma_{x y}\left(\Gamma_{0}, \Gamma_{y}\right)=\tilde{\sigma}_{x y}^{(\text {sea })}\left(\Gamma_{0}\right)+\sigma_{x y}^{\text {(surface) }}\left(\Gamma_{0}\right)+\sigma_{x y}^{(3 / 2)}\left(\Gamma_{0}, \Gamma_{y}\right) .
$$

The key player in our discussion is the new contribution

$$
\begin{aligned}
\sigma_{x y}^{(3 / 2)}= & \frac{e^{2}}{h} \frac{m}{2 \pi} \int_{-\infty}^{\infty} d k_{x} \int_{\Gamma_{0}}^{\infty} d \omega \\
& \times \operatorname{Re} \frac{\Theta\left[\mathcal{A}\left(k_{x}, \omega\right)\right]}{\left[k_{x}^{2}+m^{2}+(i \mu+\omega)^{2}\right]^{3 / 2}}, \\
\mathcal{A}\left(k_{x}, \omega\right)= & \Gamma_{y}^{2}+\mu^{2}-m^{2}-\frac{\mu^{2} \omega^{2}}{\Gamma_{y}^{2}}-\omega^{2}-k_{x}^{2} .
\end{aligned}
$$

Here, the Heaviside function $\Theta$ originates from using the residue theorem when extending the integral over $k_{y}$ to the upper complex plane. It constrains the new contribution to the Hall conductance to appear only if

$$
\epsilon:=\frac{\Gamma_{y}}{\Gamma_{0}} \sqrt{\frac{\Gamma_{y}^{2}+\mu^{2}-m^{2}}{\Gamma_{y}^{2}+\mu^{2}}}>1 .
$$

This condition can only be satisfied for $\Gamma_{y}>\Gamma_{0}$, and thus for a system with net gain in one mode. For $\mu=\Gamma_{0}=0$, we find that $\sigma_{x y}^{(3 / 2)}$ is linked to the appearance of exceptional points, since both appear for $\Gamma_{y}^{2} \geqslant m^{2}$. In general, however, exceptional points in the complex spectrum and the new contribution to the Hall conductance appear independently from one another. In particular, it is possible to have exceptional points while $\sigma_{x y}^{(3 / 2)}=0$ if $\Gamma_{0} \geqslant \Gamma_{y}$, or to have no exceptional points but nonzero $\sigma_{x y}^{(3 / 2)}$ if $\mu \neq 0$ and $\Gamma_{y} \gg \Gamma_{0}$. 

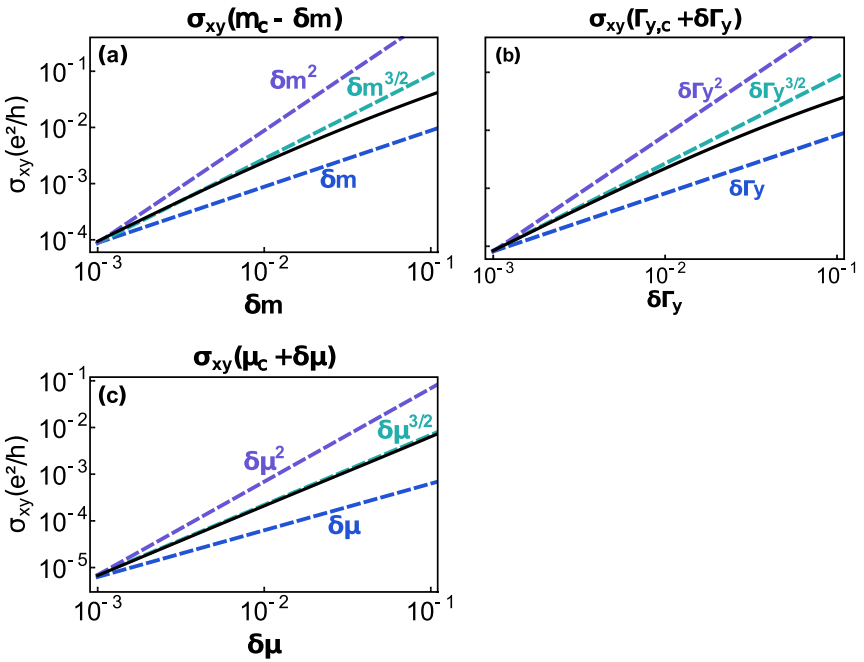

FIG. 1. The universal scaling of the Hall conductance contribution $\sigma_{x y}^{(3 / 2)}$ as a function of the system parameters $m, \Gamma_{y}$, and $\mu$. Here, $m_{c}, \Gamma_{y c}$, and $\mu_{c}$ denote, respectively, the critical values of the mass, the non-Hermiticity, and the chemical potential for which $\epsilon=1$. The exact function $\sigma_{x y}^{(3 / 2)}$ (solid line) is then compared to different power laws (dashed lines), showing that it scales indeed with an exponent $3 / 2$ as a function of these system parameters. The remaining parameters are $\mu=0.5, \Gamma_{y}=1$, and $m=1$.

To explicitly evaluate Eq. (7), we proceed by performing the integration over $k_{x}$, which yields

$$
\sigma_{x y}^{(3 / 2)}=\frac{e^{2}}{h} \frac{m}{\pi} \int_{\Gamma_{0}}^{\epsilon \Gamma_{0}} d \omega \operatorname{Re} \frac{\Theta(\epsilon-1) k_{0}(\omega)}{\left(\Gamma_{y}+i \omega \mu / \Gamma_{y}\right)\left[m^{2}+(\omega+i \mu)^{2}\right]}
$$

with $k_{0}(\omega)=\sqrt{\left(\Gamma_{y}^{2}+\mu^{2}-m^{2}\right)-\mu^{2} \omega^{2} / \Gamma_{y}^{2}-\omega^{2}}$. For $\mu=$ 0 , this integral can be performed exactly, and we find near the onset (i.e., for $\epsilon \approx 1$ ) that

$$
\sigma_{x y}^{(3 / 2)}(\mu=0) \approx \frac{2 e^{2} \sqrt{2} m}{3 \pi h} \frac{\Gamma_{y}^{2}-m^{2}}{\Gamma_{y}^{3}} \Theta(\epsilon-1)(\epsilon-1)^{3 / 2} .
$$

The contribution $\sigma_{x y}^{(3 / 2)}$ to the Hall conductance thus scales with a power of $3 / 2$ with the distance to its onset given by $\epsilon$. This nonanalytic behavior of the conductance is, however, by no means restricted to $\mu=0$. While we did not obtain a general closed result for the integral for $\mu>0$, analytic progress can be made for $\epsilon \approx 1$. We can then approximate $\omega \approx \Gamma_{0}$ and neglect terms containing $\omega-\Gamma_{0}$ in the denominator. The conductance can then be approximated as

$$
\begin{aligned}
\sigma_{x y}^{(3 / 2)} & \approx \frac{e^{2}}{h} \frac{m}{\pi} \int_{\Gamma_{0}}^{\epsilon \Gamma_{0}} d \omega \operatorname{Re} \frac{\Theta(\epsilon-1) \sqrt{\epsilon^{2} \Gamma_{0}^{2}-\omega^{2}} \sqrt{\mu^{2} / \Gamma_{y}^{2}+1}}{\left(\Gamma_{y}+i \mu \Gamma_{0} / \Gamma_{y}\right)\left[m^{2}+\left(\Gamma_{0}+i \mu\right)^{2}\right]} \\
& \propto \Theta(\epsilon-1)(\epsilon-1)^{3 / 2} .
\end{aligned}
$$

This shows that the Hall conductance generically contains a contribution that is nonanalytic in the onset parameter $\epsilon$. We confirmed this nonanalytic onset scaling of $\sigma_{x y}^{(3 / 2)}$ by evaluating Eq. (9) numerically upon variation of either the mass, the chemical potential, or the non-Hermiticity $\Gamma_{y}$. This universal scaling is depicted in Fig. 1.

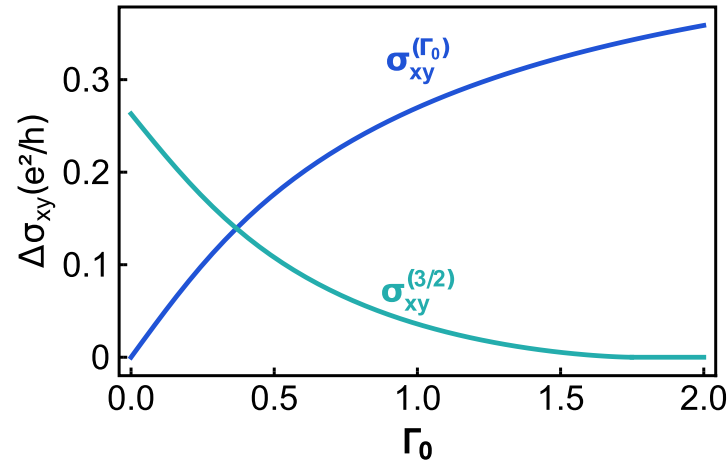

FIG. 2. Contributions of Hall conductance as a function of the global loss term $\Gamma_{0}$ in units of $e^{2} / h$, in the regime where the system experiences both loss and gain $\left(\Gamma_{y}=2\right)$, at finite chemical potential $(\mu=0.5)$. The mass is taken to be unity. $\sigma_{x y}^{\left(\Gamma_{0}\right)}=1 / 2-\left|\tilde{\sigma}_{x y}^{(\text {sea })}\right|$ is the Fermi sea contribution controlled by $\Gamma_{0}$ alone whereas $\sigma_{x y}^{(3 / 2)}$ is given in the main text.

An important practical condition for a successful detection of $\sigma_{x y}^{(3 / 2)}$ is that it is not overwhelmed by the residual non-universal Hall conductance. In particular, the total Hall conductance is dominated by its nonuniversal contributions if the constant loss term $\Gamma_{0}$ is not much smaller than $\Gamma_{y}$; see Fig. 2. The most promising setup for observing the nonanalytic contribution to the Hall conductance is thus vanishing chemical potential and compensated gains and losses (i.e., $\mu=\Gamma_{0}=0$ ). Another practical concern in open quantum systems is heating. To counter this effect, experiments should perform the Hall measurement sufficiently rapidly before the system heats up to temperatures comparable to the gap.

The nonanalytic contribution to the Hall conductance identified in this work is fundamentally different from the nonuniversality of the Hall response of effective nonHermitian Chern insulators resulting from the coupling to an environment on the level of self-energies $[15,16,58,60]$. Such kinds of effective non-Hermitian Hamiltonians have to respect the causality condition $\Gamma_{0}>\left|\Gamma_{x, y, z}\right|$. Physically, this condition encodes that self-energies are an effective description of a microscopically Hermitian system in which low-energy quasiparticles have a finite lifetime due to scattering into other states. As heralded by Eq. (8), the universal scaling identified in this work is in fact absent in such an effectively "lossy" non-Hermitian system. We thus conclude that non-Hermitian Chern insulators with $\Gamma_{0}>\left|\Gamma_{x, y, z}\right|$, in particular those deriving from complex self-energies, exhibit a fundamentally unquantized and nonuniversal Hall response. Another factor that could obstruct the observation of the 3/2-power law identified in this work would be a strong momentum dependence of the non-Hermitian terms. Although it is believed that momentum-independent gain-loss parameters describe the low-energy physics in many experimental systems, momentum-dependent non-Hermitian terms are in no way forbidden. If they dominate over the momentumindependent terms, the non-Hermitian Chern insulator will in general exhibit corrections with different power laws [15]. In open systems, however, true loss and gain are a physical reality. This is already apparent on the level of Lindblad 
master equations, for which a gain term of the form $i \Gamma_{0} \sigma_{0}+$ $i \Gamma_{y} \sigma_{y}$ results from the combination of Lindblad operators $L_{1}=\sqrt{\left(\Gamma_{0}+\Gamma_{y}\right)}\left(c_{\uparrow}-i c_{\downarrow}\right)$ and $L_{2}=\sqrt{\left(-\Gamma_{0}+\Gamma_{y}\right)}\left(c_{\uparrow}^{\dagger}-\right.$ $\left.i c_{\downarrow}^{\dagger}\right)$. The biorthogonal linear response theory we set up in the present work generalizes the discussion of non-Hermitian Hamiltonians from spectral properties to linear response functions. While the nonanalytic contribution identified in this work is independent of the topological transition at $m=0$ and of appearance of exceptional points, it is somewhat reminiscent of the critical scaling associated to topological phase transitions $[61,62]$, where the onset is related to special points in parameter space. Moreover, since impurity potential induced Anderson localization affects bulk and edge states differently in quantum Hall systems, it will be interesting to study how impurity scattering in particular affects the deviation from conductance when such systems are coupled to an environment. These two aspects will be explored in future work.

In summary, we have analyzed a two-dimensional Chern insulator coupled to an environment that induces loss and gain of quasiparticles. Starting from the corresponding nonHermitian Hamiltonian, we propose a generalized biorthogonal linear response theory for non-Hermitian Hamiltonians, and show that this scheme can be connected to a Matsubaralike formalism via analytic continuation. In Chern insulators with loss, our scheme recovers results derived in the familiar language of complex self-energies stating that the Hall conductance of a lossy Chern insulator is nonuniversal and not quantized. In addition, we also show that the Hall conductance is unaffected by the presence of exceptional points. Qualitatively new effects only emerge due to suitable combinations of loss and gain, which generally lead to the emergence of a new contribution to the Hall conductance. We demonstrated that near its onset this new contribution is nonanalytic and scales with a universal exponent $3 / 2$ as a function of all system parameters. This new form of nonanalytic behavior in open topological quantum systems is independent of the singularities found near exceptional points in the complex spectrum of the effective Hamiltonian. Our findings thereby open a new avenue for using transport as a probe for non-Hermitian topological phases.

Acknowledgments. The authors acknowledge helpful discussions with J. C. Budich. S.G. and T.L.S. acknowledge support by the National Research Fund, Luxembourg under grants ATTRACT 7556175 and PRIDE/15/10935404. T.M. acknowledges financial support by the Deutsche Forschungsgemeinschaft via the Emmy Noether Programme ME4844/11 (Project No. 327807255), the Collaborative Research Center SFB 1143 (Project No. 247310070), and the Cluster of Excellence on Complexity and Topology in Quantum Matter ct.qmat (EXC 2147, Project No. 390858490).

\section{APPENDIX A: BIORTHOGONAL LINEAR RESPONSE THEORY}

Biorthogonal quantum mechanics has been reviewed in detail in Ref. [27]. For the derivation of the Matsubara technique and linear response theory, it is sufficient to take into account that (a) there exists a unique mapping between a given right eigenstate $\left|\phi_{n}\right\rangle$ and its corresponding left eigenstate, labeled $\left|\tilde{\phi}_{n}\right\rangle$, (b) left and right eigenstates can be chosen as pairwise orthonormal, $\left\langle\tilde{\phi}_{m} \mid \phi_{n}\right\rangle=\delta_{m n}$, and (c) these states together allow a biorthogonal completeness relation $\mathbb{1}=\sum_{n}\left|\phi_{n}\right\rangle\left\langle\tilde{\phi}_{n}\right|$.

We define the correlation function of two operators $A$ and $B$ in Matsubara space as

$$
C_{A B}^{M}(\tau)=-\operatorname{Tr}^{\prime}\left[\rho T_{\tau} A(\tau) B(0)\right]
$$

where $\rho=e^{-\beta H_{\mathrm{nh}}} / Z$ is the density operator of the system, with partition function $Z=\operatorname{Tr}^{\prime} e^{-\beta H_{\mathrm{nh}}}$. Moreover, $T_{\tau}$ denotes the usual imaginary-time ordering. Importantly, the time evolution of the operators is given by $A(\tau)=e^{H_{\mathrm{nh}} \tau} A e^{-H_{\mathrm{nh}} \tau}$. These definitions make it possible to recover most results of the conventional Matsubara technique. In particular, the biorthogonal completeness relation allows a Lehmann spectral representation resulting in

$$
\begin{aligned}
C_{A B}^{M}\left(i \omega_{n}\right) & =\int_{0}^{\beta} d \tau e^{i \omega_{n} \tau} C_{A B}^{M}(\tau) \\
& =\frac{1}{Z} \sum_{m, n}\left\langle\tilde{\phi}_{m}|A| \phi_{n}\right\rangle\left\langle\tilde{\phi}_{n}|B| \phi_{m}\right\rangle \frac{e^{-\beta \epsilon_{m}}-e^{-\beta \epsilon_{n}}}{i \omega_{n}+\epsilon_{m}-\epsilon_{n}} .
\end{aligned}
$$

The aim of linear response theory is to calculate the expectation value of an operator $A$ to the first order in an external perturbation which couples to a system operator $B$. This corresponds to calculating the expectation value,

$$
\langle A\rangle(t)=\operatorname{Tr}^{\prime}[\rho(t) A],
$$

where $\rho(t)$ is the time-evolved (biorthogonal) density matrix $\rho(t)=\sum_{n} e^{-\beta \epsilon_{n}}\left|\phi_{n}(t)\right\rangle\left\langle\tilde{\phi}_{n}(t)\right|$, where the time evolution of $\left|\phi_{n}\right\rangle$ and $\left|\tilde{\phi}_{n}\right\rangle$ is given in the main text. Moreover, the external perturbation is switched on at time $t_{0}$ and leads to an additional term $V(t)=\theta\left(t-t_{0}\right) f(t) B$ in the Hamiltonian. Treating this term as a perturbation, we pass to the interaction picture as usual,

$$
\begin{aligned}
\langle A\rangle(t) & =\operatorname{Tr}^{\prime}\left[\rho_{0} A(t)\right]+\langle\delta A\rangle(t), \\
\langle\delta A\rangle(t) & =-i \int_{t_{0}}^{t} d s f(s) \operatorname{Tr}^{\prime}\left\{\rho_{0}[A(t), B(s)]\right\},
\end{aligned}
$$

where $\rho_{0}=\rho\left(t_{0}\right)$ denotes the initial state density matrix and the time dependence of the operators is governed by the unperturbed Hamiltonian, e.g., $A(t)=e^{i H_{\mathrm{nh}} t} A e^{-i H_{\mathrm{nh}} t}$. Note that the use of biorthogonal expectation values is essential to derive these equations which are formally identical to conventional linear-response theory, but allow $H_{\mathrm{nh}}$ to be non-Hermitian.

The next step is to express $\langle\delta A\rangle(t)$ using a Lehmann spectral representation based on the biorthogonal completeness relation. Then, assuming an oscillatory perturbation $f(t)=$ $e^{-i \omega_{0} t}$ and using the time evolution of the left and right eigenstates,

$$
\begin{aligned}
e^{-i H_{0} t}\left|\phi_{m}\right\rangle & =e^{-i \epsilon_{m} t}\left|\phi_{m}\right\rangle, \\
\left\langle\tilde{\phi}_{m}\right| e^{i H_{0} t} & =e^{i \epsilon_{m} t}\left\langle\tilde{\phi}_{m}\right|,
\end{aligned}
$$


makes it possible to perform the time integral, and one finds

$$
\begin{aligned}
\langle\delta A\rangle(t)= & \frac{e^{-i \omega_{0} t}}{Z} \sum_{m, n}\left\langle\tilde{\phi}_{m}|A| \phi_{n}\right\rangle\left\langle\tilde{\phi}_{n}|B| \phi_{m}\right\rangle \\
& \times \frac{e^{-\beta \epsilon_{m}}-e^{-\beta \epsilon_{n}}}{\omega_{0}+\epsilon_{m}-\epsilon_{n}}\left(1-e^{i\left(\omega_{0}+\epsilon_{m}-\epsilon_{n}\right) t}\right),
\end{aligned}
$$

where we have set $t_{0}=0$ for simplicity. Specializing for the case of the Hall conductance, one chooses $A=j_{x}$ and $B=j_{y}$ and arrives at Eq. (2) of the main text.

\section{APPENDIX B: DC HALL CONDUCTANCE FORMULA FOR GENERAL NON-HERMITIAN CHERN INSULATORS}

In this section we will outline the method to derive the DC Hall conductance of non-Hermitian Chern insulators of the form

$$
H_{\mathrm{nh}}(\mathbf{k})=\left[\mathbf{k} \cdot \boldsymbol{\sigma}+m \sigma_{z}\right]-\left(\mu+i \Gamma_{0}\right) \sigma_{0}-i \boldsymbol{\Gamma} \cdot \boldsymbol{\sigma} .
$$

This Hamiltonian is to be interpreted as the quasiparticle Hamiltonian describing a system with gain and loss. In the case of a self-energy being reduced to a non-Hermitian description, the non-Hermitian Hamiltonian is defined based on the inverse retarded Green's function. We proceed inversely and postulate a Matsubara Green's function formulation corresponding to the biorthogonal linear response in a nonHermitian Hamiltonian. In this process, the form of the self-energy needs to be chosen carefully. In the usual case, the real-time formalism is connected to the imaginary time formalism via the replacement $i \omega_{n} \rightarrow \omega \pm i 0^{+}$(for retarded and advanced Green's functions), and the self-energy is typically of the form $\Sigma\left(i \omega_{n}\right) \sim \operatorname{sgn}\left(\omega_{n}\right)$. The sign function has the analytic contination $\operatorname{sgn}\left(\omega_{n}\right) \rightarrow \operatorname{sgn}(\eta)$ for $i \omega_{n} \rightarrow \omega+i \eta$ with $\eta \rightarrow 0$, and can be interpreted as ensuring that both particle and hole excitations decay. We are interested in extending the scenario of loss, which includes the special case of a self-energy encoding a finite lifetime, to both loss and gain, and therefore define the Matsubara Green's function as

$$
\mathcal{G}(\mathbf{k}, i \omega)=\frac{1}{i \omega-\left[\mathbf{k} \cdot \boldsymbol{\sigma}+m \sigma_{z}\right]+\left[\mu+i \Gamma_{0}(i \omega)\right] \sigma_{0}+i \boldsymbol{\Gamma}(i \omega) \cdot \boldsymbol{\sigma}}, \quad \Gamma_{\mu}(i \omega):=\Gamma_{\mu} \operatorname{sgn}(\operatorname{Im} i \omega) .
$$

From now on, we use the notation $z=i \omega$. Using $\mathbf{d}=\mathbf{k}-i \boldsymbol{\Gamma}$, we find

$$
\mathcal{G}(z)=\frac{1}{z+\mu+i \Gamma_{0}(z)-\mathbf{d}(z) \cdot \boldsymbol{\sigma}}=\frac{\left[z+\mu+i \Gamma_{0}(z)\right]+\mathbf{d}(z) \cdot \boldsymbol{\sigma}}{\left[z+\mu+i \Gamma_{0}(z)\right]^{2}-\mathbf{d}(z) \cdot \mathbf{d}(z)}=\sum_{\alpha} \frac{P_{\alpha}(z)}{z+\mu+i \Gamma_{0}(z)-d_{\alpha}(z)}
$$

with $d_{ \pm}(z)= \pm d(z)$ and

$$
P_{\alpha}(\mathbf{k}, z)=\frac{1}{2}\left(1+\frac{\mathbf{d}(\mathbf{k}, z) \cdot \boldsymbol{\sigma}}{d_{\alpha}(\mathbf{k}, z)}\right), \quad d_{\alpha}(\mathbf{k}, z)=\alpha \sqrt{\left[k_{x}-i \Gamma_{x}(z)\right]^{2}+\left[k_{y}-i \Gamma_{y}(z)\right]^{2}+\left[m-i \Gamma_{z}(z)\right]^{2}} .
$$

\section{General form of the Hall conductance}

The Green's function are now expressed in terms of their spectral decomposition, whereas the derivatives of inverse Green's functions with respect to the momenta are just Pauli matrices. The derivative with respect to the Matsubara frequency is special because it involves the derivative of the sign function. (Anti)symmetrizing the Ishikawa-Matsuyama formula in units of $\hbar=e=$ 1 with respect to $x$ and $y$, the Hall conductance reads

$$
\begin{aligned}
\sigma_{x y}= & \int \frac{d^{2} k}{(2 \pi)^{2}} \int_{-i \infty}^{i \infty} \frac{d z}{2 \pi} \frac{1}{2}\left\{\operatorname{Tr}\left[\sigma_{x} P_{\alpha}(\mathbf{k}, z) \sigma_{y} P_{\beta}(\mathbf{k}, z)\left(\partial_{z} \mathcal{G}^{-1}(\mathbf{k}, z)\right) P_{\gamma}(\mathbf{k}, z)\right]-\operatorname{Tr}\left[\sigma_{y} P_{\alpha}(\mathbf{k}, z) \sigma_{x} P_{\beta}(\mathbf{k}, z)\left(\partial_{z} \mathcal{G}^{-1}(\mathbf{k}, z)\right) P_{\gamma}(\mathbf{k}, z)\right]\right\} \\
& \times \frac{1}{z+\mu+i \Gamma_{0}(z)-d_{\alpha}(\mathbf{k}, z)} \frac{1}{z+\mu+i \Gamma_{0}(z)-d_{\beta}(\mathbf{k}, z)} \frac{1}{z+\mu+i \Gamma_{0}(z)-d_{\gamma}(\mathbf{k}, z)}
\end{aligned}
$$

The inverse Green's function reads $\mathcal{G}^{-1}(\mathbf{k}, z)=\left[z+i \Gamma_{0}(z)+\right.$ $i \Gamma(z) \cdot \sigma+(\cdots)]$, where $(\cdots)$ are frequency independent terms. The derivative of the sign function is given by $\partial_{z} \operatorname{sgn}(z)=2 \delta(z)$ and hence the derivative of the inverse Green's function with respect to the Matsubara frequency gives rise to two contributions:

$$
\partial_{z} \mathcal{G}^{-1}(\mathbf{k}, z)=1+2 i \delta(z)\left(\Gamma_{0}+\boldsymbol{\Gamma} \cdot \boldsymbol{\sigma}\right) .
$$

The constant term 1 gives rise to Fermi sea contributions since it does not affect the integration over the full frequency range: it contains contribution from all the occupied states.
The second term involving the delta function only gives rise to contributions for $z=0$, that is, from the Fermi surface. We can group the Fermi surface and Fermi sea contributions, and split the DC Hall conductance into

$$
\sigma_{x y}=\sigma_{x y}^{\text {(sea) }}+\sigma_{x y}^{\text {(surface) }},
$$

where $\sigma_{x y}^{\text {(surface) }}$ is the Fermi surface contribution and $\sigma_{x y}^{\text {(sea) }}$ the (usual) Fermi sea contribution. In the absence of nonHermitian terms, the Fermi surface term vanishes and $\sigma_{x y}^{(\text {sea }}$ is the quantized Hall conductance. 


\section{Fermi surface contributions}

Using $\operatorname{sgn}(0)=0$, the Fermi surface contribution can be written as

$$
\begin{aligned}
\sigma_{x y}^{(\text {surface })}= & i \int \frac{d^{2} k}{(2 \pi)^{2}} \frac{1}{2 \pi}\left\{\operatorname{Tr}\left[\sigma_{x} P_{\alpha}(\mathbf{k}) \sigma_{y} P_{\beta}(\mathbf{k})\left(\Gamma_{0} \sigma_{0}+\boldsymbol{\Gamma} \cdot \boldsymbol{\sigma}\right) P_{\gamma}(\mathbf{k})\right]-\operatorname{Tr}\left[\sigma_{y} P_{\alpha}(\mathbf{k}) \sigma_{x} P_{\beta}(\mathbf{k})\left(\Gamma_{0} \sigma_{0}+\boldsymbol{\Gamma} \cdot \boldsymbol{\sigma}\right) P_{\gamma}(\mathbf{k})\right]\right\} \\
& \times \frac{1}{\mu-d_{\alpha}(\mathbf{k})} \frac{1}{\mu-d_{\beta}(\mathbf{k})} \frac{1}{\mu-d_{\gamma}(\mathbf{k})}
\end{aligned}
$$

where the projector now takes on the simpler form

$$
P_{\alpha}(k)=\frac{1}{2}\left(1+\frac{\mathbf{d} \cdot \boldsymbol{\sigma}}{d_{\alpha}(\mathbf{k})}\right), \quad \mathbf{d}(\mathbf{k})=\left(k_{x}, k_{y}, m\right), \quad d_{\alpha}(\mathbf{k})=\alpha \sqrt{k^{2}+m^{2}} .
$$

In the trace, contributions from $\Gamma_{x} \sigma_{x}$ and $\Gamma_{y} \sigma_{y}$ are zero due to the antisymmetry of the conductance tensor. Hence, only terms $\propto \Gamma_{0}$ and $\Gamma_{z}$ will contribute to the trace. We can thus write $\sigma_{x y}^{\text {(surface) }}$ as the sum of two terms,

$$
\sigma_{x y, a}^{(\text {surface })}=i \Gamma_{0} \int \frac{d^{2} k}{(2 \pi)^{2}} \frac{1}{2 \pi}\left\{\operatorname{Tr}\left[\sigma_{x} P_{\alpha}(\mathbf{k}) \sigma_{y} P_{\beta}(\mathbf{k}) P_{\gamma}(\mathbf{k})\right]-\operatorname{Tr}\left[\sigma_{y} P_{\alpha}(\mathbf{k}) \sigma_{x} P_{\beta}(\mathbf{k}) P_{\gamma}(\mathbf{k})\right]\right\} \frac{1}{\mu-d_{\alpha}(\mathbf{k})} \frac{1}{\mu-d_{\beta}(\mathbf{k})} \frac{1}{\mu-d_{\gamma}(\mathbf{k})}
$$

and

$$
\sigma_{x y, b}^{(\text {surface })}=i \Gamma_{z} \int \frac{d^{2} k}{(2 \pi)^{2}} \frac{1}{2 \pi}\left\{\operatorname{Tr}\left[\sigma_{x} P_{\alpha}(\mathbf{k}) \sigma_{y} P_{\beta}(\mathbf{k}) \sigma_{z} P_{\gamma}(\mathbf{k})\right]-\operatorname{Tr}\left[\sigma_{y} P_{\alpha}(\mathbf{k}) \sigma_{x} P_{\beta}(\mathbf{k}) \sigma_{z} P_{\gamma}(\mathbf{k})\right]\right\} \frac{1}{\mu-d_{\alpha}(\mathbf{k})} \frac{1}{\mu-d_{\beta}(\mathbf{k})} \frac{1}{\mu-d_{\gamma}(\mathbf{k})} .
$$

Performing the trace on both expressions, we find

$$
\begin{aligned}
\sigma_{x y, a}^{\text {(surface) }} & =-\frac{4}{2 \pi} \Gamma_{0} \int \frac{d^{2} k}{(2 \pi)^{2}} \frac{m}{\left[\left(k^{2}+m^{2}\right)-\mu^{2}\right]^{2}}=-\frac{1}{(2 \pi)^{2}} \frac{2 m \Gamma_{0}}{m^{2}-\mu^{2}}, \\
\sigma_{x y, b}^{\text {(surface) }} & =-\frac{4}{2 \pi} \Gamma_{z} \int \frac{d^{2} k}{(2 \pi)^{2}} \frac{\mu}{\left[\left(k^{2}+m^{2}\right)-\mu^{2}\right]^{2}}=-\frac{1}{(2 \pi)^{2}} \frac{2 \mu \Gamma_{z}}{m^{2}-\mu^{2}} .
\end{aligned}
$$

Hence, the result of this contribution reads, retrieving units,

$$
\sigma_{x y}^{(\text {surface })}=-\frac{1}{\pi} \frac{e^{2}}{h} \frac{\mu \Gamma_{z}+m \Gamma_{0}}{m^{2}-\mu^{2}} .
$$

\section{Fermi sea contributions}

Evaluating the trace, the Fermi sea contribution can be brought to the form

$$
\sigma_{x y}^{(\text {sea })}=\int \frac{d^{2} k}{(2 \pi)^{2}} \int_{-i \infty}^{i \infty} \frac{d z}{2 \pi} \frac{1}{2} \frac{4 i\left[m-i \Gamma_{z}(z)\right]}{\left[d^{2}(\mathbf{k}, z)-\left\{z+i \Gamma_{0}(z)+\mu\right\}^{2}\right]^{2}} .
$$

Defining $\varepsilon_{ \pm}(\mathbf{k})=\sqrt{\left(k_{x} \mp i \Gamma_{x}\right)^{2}+\left(k_{y} \mp i \Gamma_{y}\right)^{2}+\left(m \mp i \Gamma_{z}\right)^{2}}$, we obtain

$$
\sigma_{x y}^{(\text {sea })}=2 i \int \frac{d^{2} k}{(2 \pi)^{2}} \int_{i 0^{+}}^{+i \infty} \frac{d z}{2 \pi}\left\{\frac{\left(m+i \Gamma_{z}\right)}{\left[\varepsilon_{-}^{2}(\mathbf{k})-\left(z+i \Gamma_{0}-\mu\right)^{2}\right]^{2}}+\frac{\left(m-i \Gamma_{z}\right)}{\left[\varepsilon_{+}^{2}(\mathbf{k})-\left(z+i \Gamma_{0}+\mu\right)^{2}\right]^{2}}\right\} .
$$

The two terms can be interpreted as contributions from the conduction band and valence band of the system. The information about dissipation and the interaction is contained in the $\varepsilon_{ \pm}(\mathbf{k})$ terms. Specializing to the case $\Gamma_{y} \neq 0$ but $\Gamma_{x}=0=\Gamma_{z}$, and with $z=i \omega$, we obtain

$$
\sigma_{x y}^{(\text {sea })}=-2 m \int \frac{d k_{x}}{(2 \pi)^{2}} \int_{0}^{\infty} \frac{d \omega}{2 \pi} \sum_{\alpha= \pm} \int d k_{y} \frac{1}{\left[\left(k_{y}-i \Gamma_{y}\right)^{2}+\left[k_{x}^{2}+m^{2}+\left(\alpha i \mu+\Gamma_{0}+\omega\right)^{2}\right]\right]^{2}} .
$$

The $k_{y}$ integrals can be evaluated using the residue theorem, which yields

$$
\sigma_{x y}^{(\text {sea })}=-m \int \frac{d k_{x}}{(2 \pi)^{2}} \int_{0}^{\infty} d \omega \operatorname{Re} \frac{1}{{\sqrt{k_{x}^{2}+m^{2}+\left(i \mu+\Gamma_{0}+\omega\right)^{2}}}^{3}} \Theta\left[-\mathcal{A}\left(k_{x}, \omega+\Gamma_{0}\right)\right] .
$$

Using $\Theta(-x)=1-\Theta(x)$, we decompose $\sigma_{x y}^{(\text {sea })}$ as a contribution $\tilde{\sigma}_{x y}^{(\text {sea })}$ without phase-space restriction minus a contribution which corresponds to the conductance contribution $\sigma^{(3 / 2)}$ discussed in the main text,

$$
\sigma_{x y}^{(\text {sea })}=\tilde{\sigma}_{x y}^{(\text {sea })}+\sigma_{x y}^{(3 / 2)}
$$


where

$$
\tilde{\sigma}_{x y}^{(\text {sea })}=-\frac{m}{|m|} \frac{e^{2}}{h} \frac{1}{2 \pi}\left[\frac{\pi}{2}-\arctan \left(\frac{\Gamma_{0}^{2}+\mu^{2}-m^{2}}{2|m| \Gamma_{0}}\right)\right],
$$

as already derived in Refs. $[15,16]$ and $\sigma_{x y}^{(3 / 2)}$ is given, after a shift of variable $\omega+\Gamma_{0} \rightarrow \omega$ in the main text.

[1] J. M. Kosterlitz and D. J. Thouless, Ordering, metastability and phase transitions in two-dimensional systems, J. Phys. C: Solid State Phys. 6, 1181 (1973).

[2] X.-G. Wen, Colloquium: Zoo of quantum-topological phases of matter, Rev. Mod. Phys. 89, 041004 (2017).

[3] C.-K. Chiu, J. C. Y. Teo, A. P. Schnyder, and S. Ryu, Classification of topological quantum matter with symmetries, Rev. Mod. Phys. 88, 035005 (2016).

[4] X.-L. Qi and S.-C. Zhang, Topological insulators and superconductors, Rev. Mod. Phys. 83, 1057 (2011).

[5] A. Kitaev, V. Lebedev, and M. Feigel'man, Periodic table for topological insulators and superconductors, in Advances in Theoretical Physics: Landau Memorial Conference, edited by V. Lebedev and M. Feigelman, AIP Conf. Proc. No. 1134 (AIP, New York, 2009), p. 22.

[6] D. J. Thouless, M. Kohmoto, M. P. Nightingale, and M. den Nijs, Quantized Hall Conductance in a Two-Dimensional Periodic Potential, Phys. Rev. Lett. 49, 405 (1982).

[7] F. D. M. Haldane, Model for a Quantum Hall Effect without Landau Levels: Condensed-Matter Realization of the "Parity Anomaly”, Phys. Rev. Lett. 61, 2015 (1988).

[8] C.-X. Liu, S.-C. Zhang, and X.-L. Qi, The quantum anomalous Hall effect: Theory and experiment, Annu. Rev. Condens. Matter Phys. 7, 301 (2016).

[9] T. Gulden, M. Janas, Y. Wang, and A. Kamenev, Universal Finite-Size Scaling around Topological Quantum Phase Transitions, Phys. Rev. Lett. 116, 026402 (2016).

[10] B. Zhou, H.-Z. Lu, R.-L. Chu, S.-Q. Shen, and Q. Niu, Finite Size Effects on Helical Edge States in a Quantum Spin-Hall System, Phys. Rev. Lett. 101, 246807 (2008).

[11] S. M. Albrecht, A. P. Higginbotham, M. Madsen, F. Kuemmeth, T. S. Jespersen, J. Nygård, P. Krogstrup, and C. M. Marcus, Exponential protection of zero modes in Majorana islands, Nature (London) 531, 206 (2016).

[12] G. G. Giusteri, F. Mattiotti, and G. L. Celardo, Non-Hermitian Hamiltonian approach to quantum transport in disordered networks with sinks: Validity and effectiveness, Phys. Rev. B 91, 094301 (2015).

[13] E. J. Bergholtz, J. C. Budich, and F. K. Kunst, Exceptional topology of non-Hermitian systems, Rev. Mod. Phys. 93, 015005 (2021).

[14] L. E. F. F. Torres, Perspective on topological states of nonHermitian lattices, J. Phys. Mater. 3, 014002 (2019).

[15] T. M. Philip, M. R. Hirsbrunner, and M. J. Gilbert, Loss of Hall conductivity quantization in a non-Hermitian quantum anomalous Hall insulator, Phys. Rev. B 98, 155430 (2018).

[16] M. R. Hirsbrunner, T. M. Philip, and M. J. Gilbert, Topology and observables of the non-Hermitian Chern insulator, Phys. Rev. B 100, 081104(R) (2019).

[17] M. Berry, Physics of nonhermitian degeneracies, Czech. J. Phys. 54, 1039 (2004).
[18] I. Rotter, A non-Hermitian Hamilton operator and the physics of open quantum systems, J. Phys. A: Math. Theor. 42, 153001 (2009).

[19] H. Shen, B. Zhen, and L. Fu, Topological Band Theory for NonHermitian Hamiltonians, Phys. Rev. Lett. 120, 146402 (2018).

[20] M. Papaj, H. Isobe, and L. Fu, Nodal arc of disordered Dirac fermions and non-Hermitian band theory, Phys. Rev. B 99, 201107(R) (2019).

[21] S. Diehl, E. Rico, M. A. Baranov, and P. Zoller, Topology by dissipation in atomic quantum wires, Nat. Phys. 7, 971 (2011).

[22] R. El-Ganainy, K. G. Makris, M. Khajavikhan, Z. H. Musslimani, S. Rotter, and D. N. Christodoulides, NonHermitian physics and PT symmetry, Nat. Phys. 14, 11 (2018).

[23] F. Song, S. Yao, and Z. Wang, Non-Hermitian Skin Effect and Chiral Damping in Open Quantum Systems, Phys. Rev. Lett. 123, 170401 (2019).

[24] M. Naghiloo, M. Abbasi, Y. N. Joglekar, and K. W. Murch, Quantum state tomography across the exceptional point in a single dissipative qubit, Nat. Phys. 15, 1232 (2019).

[25] F. Minganti, A. Miranowicz, R. W. Chhajlany, and F. Nori, Quantum exceptional points of non-Hermitian Hamiltonians and Liouvillians: The effects of quantum jumps, Phys. Rev. A 100, 062131 (2019).

[26] V. Tripathi and V. M. Vinokur, $\mathcal{P} \mathcal{T}$-symmetric effective model for nonequilibrium phase transitions in a dissipative fermionic Mott insulator chain, Sci. Rep. 10, 7304 (2020).

[27] D. C. Brody, Biorthogonal quantum mechanics, J. Phys. A: Math. Theor. 47, 035305 (2013).

[28] L. Herviou, N. Regnault, and J. H. Bardarson, Entanglement spectrum and symmetries in non-Hermitian fermionic noninteracting models, SciPost Phys. 7, 69 (2019).

[29] S. Wimberger, C. A. Parra-Murillo, and G. Kordas, NonHermitian approach to decaying ultracold bosonic systems, J. Phys. Conf. Ser. 442, 012029 (2013).

[30] J. Li, A. K. Harter, J. Liu, L. de Melo, Y. N. Joglekar, and L. Luo, Observation of parity-time symmetry breaking transitions in a dissipative Floquet system of ultracold atoms, Nat. Commun. 10, 855 (2019).

[31] W. Gou, T. Chen, D. Xie, T. Xiao, T.-S. Deng, B. Gadway, W. Yi, and B. Yan, Tunable Nonreciprocal Quantum Transport through a Dissipative Aharonov-Bohm Ring in Ultracold Atoms, Phys. Rev. Lett. 124, 070402 (2020).

[32] Y. Xu, S.-T. Wang, and L.-M. Duan, Weyl Exceptional Rings in a Three-Dimensional Dissipative Cold Atomic Gas, Phys. Rev. Lett. 118, 045701 (2017).

[33] L. Pan, S. Chen, and X. Cui, Interacting non-Hermitian ultracold atoms in a harmonic trap: Two-body exact solution and a high-order exceptional point, Phys. Rev. A 99, 063616 (2019).

[34] L. Li, C. H. Lee, and J. Gong, Topological Switch for NonHermitian Skin Effect in Cold-Atom Systems with Loss, Phys. Rev. Lett. 124, 250402 (2020). 
[35] J. Wang, F. Li, and X. X. Yi, Hall conductance of a nonHermitian two-band system with k-dependent decay rates, arXiv:2101.02393.

[36] A. Regensburger, C. Bersch, M.-A. Miri, G. Onishchukov, D. N. Christodoulides, and U. Peschel, Parity-time synthetic photonic lattices, Nature (London) 488, 167 (2012).

[37] B. Zhen, C. W. Hsu, Y. Igarashi, L. Lu, I. Kaminer, A. Pick, S.-L. Chua, J. D. Joannopoulos, and M. Soljačić, Spawning rings of exceptional points out of Dirac cones, Nature (London) 525, 354 (2015).

[38] C. Hahn, Y. Choi, J. W. Yoon, S. H. Song, C. H. Oh, and P. Berini, Observation of exceptional points in reconfigurable non-hermitian vector-field holographic lattices, Nat. Commun. 7, 12201 (2016).

[39] A. Cerjan, S. Huang, M. Wang, K. P. Chen, Y. Chong, and M. C. Rechtsman, Experimental realization of a Weyl exceptional ring, Nat. Photonics 13, 623 (2019).

[40] Ş. K. Özdemir, S. Rotter, F. Nori, and L. Yang, Parity-time symmetry and exceptional points in photonics, Nat. Mater. 18, 783 (2019).

[41] M.-A. Miri and A. Alù, Exceptional points in optics and photonics, Science 363, eaar7709 (2019).

[42] Y. Huang, Y. Shen, C. Min, S. Fan, and G. Veronis, Unidirectional reflectionless light propagation at exceptional points, Nanophotonics 6, 977 (2017).

[43] A. Guo, G. J. Salamo, D. Duchesne, R. Morandotti, M. VolatierRavat, V. Aimez, G. A. Siviloglou, and D. N. Christodoulides, Observation of $\mathcal{P} \mathcal{T}$-Symmetry Breaking in Complex Optical Potentials, Phys. Rev. Lett. 103, 093902 (2009).

[44] H. Hodaei, A. U. Hassan, S. Wittek, H. Garcia-Gracia, R. El-Ganainy, D. N. Christodoulides, and M. Khajavikhan, Enhanced sensitivity at higher-order exceptional points, Nature (London) 548, 187 (2017).

[45] G. Jotzu, M. Messer, R. Desbuquois, M. Lebrat, T. Uehlinger, D. Greif, and T. Esslinger, Experimental realization of the topological Haldane model with ultracold fermions, Nature (London) 515, 237 (2014).

[46] S. Nakajima, T. Tomita, S. Taie, T. Ichinose, H. Ozawa, L. Wang, M. Troyer, and Y. Takahashi, Topological Thouless pumping of ultracold fermions, Nat. Phys. 12, 296 (2016).
[47] K. Ishikawa and T. Matsuyama, Magnetic field induced multicomponent QED3 and quantum Hall effect, Z. Phys. C 33, 41 (1986).

[48] K. Ishikawa and T. Matsuyama, A microscopic theory of the quantum hall effect, Nucl. Phys. B 280, 523 (1987).

[49] N. Imai, K. Ishikawa, T. Matsuyama, and I. Tanaka, Field theory in a strong magnetic field and the quantum Hall effect: Integer Hall effect, Phys. Rev. B 42, 10610 (1990).

[50] Z. Wang, X.-L. Qi, and S.-C. Zhang, Topological Order Parameters for Interacting Topological Insulators, Phys. Rev. Lett. 105, 256803 (2010).

[51] Q. Niu, D. J. Thouless, and Y.-S. Wu, Quantized Hall conductance as a topological invariant, Phys. Rev. B 31, 3372 (1985).

[52] V. Gurarie, Single-particle Green's functions and interacting topological insulators, Phys. Rev. B 83, 085426 (2011).

[53] W. Chen, Weakly interacting topological insulators: Quantum criticality and the renormalization group approach, Phys. Rev. B 97, 115130 (2018).

[54] A. Tsvelik, Quantum Field Theory in Condensed Matter Physics (Cambridge University Press, Cambridge New York, 2003).

[55] A. Altland, Condensed Matter Field Theory (Cambridge University Press, Leiden, 2010).

[56] A. Belavin, A. Polyakov, A. Schwartz, and Y. Tyupkin, Pseudoparticle solutions of the Yang-Mills equations, Phys. Lett. B 59, 85 (1975).

[57] S.-S. Chern and J. Simons, Characteristic forms and geometric invariants, Ann. Math 99, 48 (1974).

[58] Y. Chen and H. Zhai, Hall conductance of a non-Hermitian Chern insulator, Phys. Rev. B 98, 245130 (2018).

[59] M. Grifoni and P. Hänggi, Driven quantum tunneling, Phys. Rep. 304, 229 (1998).

[60] C. Wang and X. R. Wang, Non-quantized edge channel conductance and zero conductance fluctuation in non-Hermitian Chern insulators, arXiv:1901.06982.

[61] W. Chen, M. Legner, A. Rüegg, and M. Sigrist, Correlation length, universality classes, and scaling laws associated with topological phase transitions, Phys. Rev. B 95, 075116 (2017).

[62] W. Chen and A. P. Schnyder, Universality classes of topological phase transitions with higher-order band crossing, New J. Phys. 21, 073003 (2019). 